\title{
Quantum (not frustrated) theory of the total internal reflection as the source of the Goos-Hänchen shift
}

\author{
Jacek Jakiel ${ }^{\mathrm{a}}$ and Wiesław Kantor \\ The Henryk Niewodniczański Institute of Nuclear Physics, Polish Academy of Sciences, ul.Radzikowskiego 152, \\ 31-342 Kraków, Poland
}

\author{
Received 28 February 2014 / Received in final form 2 June 2014 \\ Published online 21 October 2014 \\ (C) The Author(s) 2014. This article is published with open access at Springerlink.com
}

\begin{abstract}
The frustrated total internal reflection theories (FTIR) from previous century are thoroughly recalculated from the, so called, monodromy operator's point of view - a theory lunched by Born and Wolf [Principles of Optics (Pergamon Press, 1975), Chap. 1.6] and Arnold [Geometric Methods in the Theory of Ordinary Differential Equations (Springer, 1987)]. Monodromy is a theory of simultaneous solution (for both reflection and transmission amplitudes) of one dimensional Schrödinger equation (for the wavefunction and its derivative) and the Maxwell equation (for electric and magnetic fields). Introducing new quantities: the dwell distance and the phase distance, we get general Goos-Hänchen (G-H) shift formula for optical tunneling for three layer system with refraction indexes $n_{0}, n_{1}, n_{2}$. This formula reduces itself to expressions known from the scientific literature for infinite air gap (infinite width of second layer). Extension to many layers is possible.
\end{abstract}

\section{Introduction to monodromy}

The phenomenon of the penetration of the light into the second medium when total internal reflection occurs was investigated first by Newton (1642-1726) and the mathematical description was given by Fresnel (1788-1827). Thereafter in the next centuries or nearly one hundred years later the total internal reflection was studied in Hall [1], Försteling [2] and Arzelies [3] papers. Leurgans and Turner [4] called the process 'frustrated', when the wave undergoing the total reflection at the first interface between two media with refractive indexes respectively $n_{0}$ and $n_{1}$ is transmitting energy to the third medium with refractive index $n_{2}$. The discovery of the Goos-Hänchen shift $[5,6]$ in 1947 (i.e. reflected beam emerges at place different then the point of incidence) caused some controversy. To solve that problem, Renard in reference [7] wrote: "Artmann... starting from the Fresnel-Maxwell equations, considered only the mathematical expressions for the incident and totally reflected beams. From the difference of phase between these two beams he was able to account for the observed shift"... The shift was different for polarization parallel and perpendicular to the plane. According to Renard "the G-H shift is identified with a translation of beam and the principle of energy conservation is used to establish the quantitative expression for

\footnotetext{
${ }^{a}$ e-mail: jacek.jakiel@ifj.edu.pl
}

the shift"... This however had been done without studying influence of middle medium width on the shift.

The FTIR phenomenon for three layer system in optics can be accurately solved through extending the Schrödinger equation monodromy operator theory on the electromagnetic wave propagation in a stratified medium.

Our calculations allow us to combine Artmann and Renard methods with not too rigorous analysis of Court and von Willisen [8].

The stratified medium corresponds to the barrier system in nuclear (or solid state) physics. The barrier system can be described by a piecewise potential what requires a piecewise-defined function. Each subfunction built on subdomain (interval) must include two possible ways of (lossless) scattering: reflection and transmission. In case of $n$ barriers the wavefunction domain is divided into $n+2$ intervals.

It is well known that second order linear differential equation has two linearly independent basic solutions i.e. regular or irregular at origin $\left(\Psi_{r}^{(j)}(x), \Psi_{i r}^{(j)}(x)\right) \equiv$ $\left(F_{l}^{(j)}(\eta, x), G_{l}^{(j)}(\eta, x)\right) \stackrel{l=0, \eta=0}{\longrightarrow}\left(\sin ^{(j)}(x), \cos ^{(j)}(x)\right)$ (e.g. see $[9,10])$. It is no unique choice. We can take also inward and outward wavefunctions $\left(\Psi_{i n}^{(j)}, \Psi_{\text {out }}^{(j)}\right)$. We assume that these solutions are known in each subdomain (interval) of the independent variable $x$. Then any solutions of the Schrödinger equation on $j$ th interval can be written as: $\psi^{(j)}(x)=A_{j} \Psi_{r}^{(j)}(x)+B_{j} \Psi_{i r}^{(j)}(x)=C_{j} \Psi_{\text {in }}^{(j)}(x)+D_{j} \Psi_{\text {out }}^{(j)}(x)$. 
The unimodular (unitary) transformation

$$
U=\left[\begin{array}{cc}
\frac{1}{\sqrt{2}} & \frac{1}{\sqrt{2}} \\
-\frac{i}{\sqrt{2}} & \frac{i}{\sqrt{2}}
\end{array}\right], \rightarrow U^{-1}=\left[\begin{array}{cc}
\frac{1}{\sqrt{2}} & \frac{i}{\sqrt{2}} \\
\frac{1}{\sqrt{2}} & -\frac{i}{\sqrt{2}}
\end{array}\right]
$$

joins both pairs of independent solutions expressed in terms of wronskian matrices:

$$
\left[W_{(\text {in }, \text { out })}^{(j)}(x)\right]_{x \in\left(x_{j}, x_{j+1}\right)}=\left[W_{(r, i r)}^{(j)}(x)\right]_{x \in\left(x_{j}, x_{j+1}\right)} \circ U
$$

where

$$
\left[W_{(r, i r)}^{(j)}(x)\right]_{x \in\left(x_{j}, x_{j+1}\right)}=\left[\begin{array}{cc}
\Psi_{r, l}^{\prime(j)}(x) & \Psi_{i r, l}^{\prime(j)}(x) \\
\Psi_{r, l}^{(j)}(x) & \Psi_{i r, l}^{(j)}(x)
\end{array}\right]_{x \in\left(x_{j}, x_{j+1}\right)}
$$

and the prime ( $I$ ) denotes derivative over $\kappa x$. Simultaneous interchange of rows and columns to get standard wronskian's matrix does not influence calculation.

We can also introduce $\Phi_{(\text {in }, \text { out })}^{(j)}(x)$ wavefunction matrix which is similar to wronskian $W_{(r, i r)}^{(j)}(x)$

$$
\left[\Phi_{(\text {in }, \text { out })}^{(j)}(x)\right]_{x \in\left(x_{j}, x_{j+1}\right)}=[U]^{-1}\left[W_{(r, i r)}^{(j)}(x)\right]_{x \in\left(x_{j}, x_{j+1}\right)}[U]
$$

$\Phi_{(\text {in }, \text { out })}^{(j)}(x)$ describes propagation of wavefunctions in a given layer (i.e. under a barrier or in a well).

To solve Schrödinger equation describing evolution of a particle through piecewise potential, without boundary condition at origin, we need to find transition matrix between input coefficients $\left(A_{0}, B_{0}\right)$ or $\left(C_{0}, D_{0}\right)$ of the wavefunction $\Phi^{(0)}$ and output ones $\left(A_{n+1}, B_{n+1}\right)$ or $\left(C_{n+1}, D_{n+1}\right)$ of the wavefunction $\Phi^{(n+1)}$. In other words, from the piecewise differentiable wavefunctions we want to build continuously differentiable solution in the whole domain. Two port network is analogous to such matrix in communication and electronic engineering. Here we have kind of quadruple quantum network

$$
\left[\begin{array}{c}
C_{n+1} \\
D_{n+1}
\end{array}\right]=\left[\begin{array}{c}
\text { Transmission } \\
\text { matrix }
\end{array}\right]\left[\begin{array}{c}
C_{0} \\
D_{0}
\end{array}\right]
$$

This transmission matrix is also a quantum tunneling analog of a multistepped transmission line.

The stationary Schrödinger equation with multipoint boundary conditions can be solved with help of monodromy operator [11]. The linear operator which relates pairs of the Schrödinger equation independent solutions before the interface (potential step) with another pair of the same kind of independent solutions behind this interface is the Schrödinger equation monodromy operator for that interface (step).

From mathematical point of view the following words are interchangeable:

- the potential step $\leftrightarrow$ interface;

- rectangular potential barrier (or a well) $\leftrightarrow$ layer.
Two potential steps form barrier (or a well), layer between two other layers is a barrier (or a well) between two physical media.

Born and Wolf [12] reduce TE and TM wave propagation in a stratified medium to a pair of telegrapher equation. The magnetic wave is proportional to the electric wave derivative. This property not important to Born and Wolf, from mathematical point of view enable us to introduce the electromagnetic monodromy operator. Analysis show that $T E$ and $T M$ wavefunction propagation through multilayer system can be described in terms of the Schrödinger multibarrier scattering.

The general form of one dimensional equation solutions the Schrödinger equation (the wavefunction and its derivative) and the Maxwell equation (the electric and magnetic fields), for a barrier (a well) system or multilayer system in case of plane waves is given by:

$$
\begin{gathered}
{\left[\begin{array}{c}
T e^{i k_{f} b} \\
0
\end{array}\right]=[M]_{f, i n}\left[\begin{array}{c}
e^{i k_{i n} a} \\
R e^{-i k_{i n} a}
\end{array}\right]} \\
{\left[\begin{array}{c}
T \\
0
\end{array}\right]=\left[\begin{array}{cc}
e^{-i k_{f} b} & 0 \\
0 & e^{i k_{f} b}
\end{array}\right][M]_{f, i n}\left[\begin{array}{cc}
e^{i k_{i n} a} & 0 \\
0 & e^{-i k_{i n} a}
\end{array}\right]\left[\begin{array}{l}
1 \\
R
\end{array}\right]}
\end{gathered}
$$

where $C_{0}=1, D_{0}=R$ and $C_{n+1}=T, D_{n+1}=0$.

If we are able to build the matrix $[M]_{f, i n}$ then it is easy to calculate unknown quantities: reflection $R$ and transmission $T$ amplitudes. Both amplitudes can be expressed as elements of that matrix. $k_{i n}$ is momentum in front of barrier or in the first layer. $k_{f}$ is wave momentum outside barrier or in the last (third) layer. The matrix $[M]_{f, \text { in }}$ is called the unimodular monodromy operator, $\operatorname{det}[M]=1$, and with accordance to the idea written by Arnold in reference [11] in our case is:

$$
[M]=\left[\begin{array}{ll}
M_{11} & M_{12} \\
M_{21} & M_{22}
\end{array}\right] ; \quad M_{12}^{*}=M_{21} ; \quad M_{11}^{*}=M_{22}
$$

what can be rewritten as composition of unitary and hermitian matrix:

$$
[M]=[M]_{U}[M]_{H}
$$

or using the unknown quantities $T=|T| e^{i \phi_{T}}, \quad R=$ $|R| e^{i \phi_{R}}$ as:

$$
[M]=\left[\begin{array}{cc}
\frac{1}{T^{*}} & -\frac{R^{*}}{T^{*}} \\
-\frac{R}{T} & \frac{1}{T}
\end{array}\right]
$$

That representation is only true for plane waves, for single potential step as well as multistep composition in case of the Schrödinger equation and for superposition of reflection and transmission amplitudes at multi layer interfaces. For example, in case of one barrier (two steps) and three 
layers we get:

$$
\begin{aligned}
{[M] } & =\left[\begin{array}{cc}
\frac{1}{T_{2}^{*}} & -\frac{R_{2}^{*}}{T_{2}^{*}} \\
-\frac{R_{2}}{T_{2}} & \frac{1}{T_{2}}
\end{array}\right]\left[\begin{array}{cc}
e^{i \varphi} & 0 \\
0 & e^{-i \varphi}
\end{array}\right]\left[\begin{array}{cc}
\frac{1}{T_{1}^{*}} & -\frac{R_{1}^{*}}{T_{1}^{*}} \\
-\frac{R_{1}}{T_{1}} & \frac{1}{T_{1}}
\end{array}\right] \\
& =\left[\begin{array}{ll}
\frac{1}{T_{1}^{*} T_{2}^{*}} e^{i \varphi}+\frac{R_{1} R_{2}^{*}}{T_{1} T_{2}^{*}} e^{-i \varphi} & -\frac{R_{1}^{*}}{T_{1}^{*} T_{2}^{*}} e^{i \varphi}-\frac{R_{2}^{*}}{T_{1} T_{2}^{*}} e^{-i \varphi} \\
-\frac{R_{2}}{T_{1}^{*} T_{2}} e^{i \varphi}-\frac{R_{1}}{T_{1} T_{2}} e^{-i \varphi} & \frac{1}{T_{1} T_{2}} e^{-i \varphi}+\frac{R_{1}^{*} R_{2}}{T_{1}^{*} T_{2}} e^{i \varphi}
\end{array}\right]
\end{aligned}
$$

where $\varphi$ is phase increment in the well or in the middle layer. In case of the barrier or FTIR, $\varphi=i \kappa L$ is pure imaginary. The middle matrix describes the evolution of sub-barrier wavefunction. This matrix has evanescent and anti-evanescent wavefunctions. Solving equations (2) and (3) we get $R=M_{21} / M_{22}$ or $T=1 / M_{22}$ :

$$
\begin{aligned}
& M_{21}=\frac{R}{T}=\frac{R_{2} T_{1} e^{i \varphi}+R_{1} T_{1}^{*} e^{-i \varphi}}{\left|T_{1}\right|^{2} T_{2}} \\
& M_{22}=\frac{1}{T}=\frac{T_{1}^{*} e^{-i \varphi}+R_{1}^{*} T_{1} R_{2} e^{i \varphi}}{\left|T_{1}\right|^{2} T_{2}} \\
& |R|^{2}=\frac{\left(R_{1}-R_{2} e^{-2 \kappa L}\right)\left(R_{1}^{*}-R_{2}^{*} e^{-2 \kappa L}\right)}{\left(1-R_{1}^{*} R_{2} e^{-2 \kappa L}\right)\left(1-R_{1} R_{2}^{*} e^{-2 \kappa L}\right)} .
\end{aligned}
$$

Calculating the monodromy operator for certain physical system i.e. barrier composition for reflected and transmitted particle, we are able to find relevant solution for analog optical system $(T E, T M)$. It is not easy though to calculate $R_{j}$ and $T_{j}$ for FTIR not applying monodromy. Additionally the reflection and refraction on two single interfaces, in case of a thin layer between them cannot be treated as independent. Probably that independence is source of the term 'frustrated'. There is no comparison of that problem to quantum mechanics in scientific literature.

It is advisable to compare expression (4)-(8) with (57)-(59) (77)-(79) in chap. 1.6 in reference [12]. There is certain difference between them: $r_{12}$ must be complex and in denominator (8) there is $r_{12}^{*}=R_{1}^{*}$. Moreover from quantum point of view $\left|r_{12}\right| \leq 1$ and $\left|r_{23}\right| \leq 1$.

Now we introduce general method of photon lossless propagation through $n$ layer. Photon undergo only reflection or refraction in interface system like the quantum particle which is scattered on the barrier system as in Figure 1.

One step transmission represented by the matrix $\mathcal{I}_{j+1}$ is the base for calculations. Action of that matrix is shown in Figure 2. Let us enumerate steps or interfaces with natural numbers starting from one. Then first step is between zero layer and first layer and the matrix $\mathcal{I}_{j+1}$ is:

$$
\mathcal{I}_{j+1}=\left[\begin{array}{cc}
\sqrt{\frac{\kappa_{j+1}}{\kappa_{j}}} & 0 \\
0 & \sqrt{\frac{\kappa_{j}}{\kappa_{j+1}}}
\end{array}\right] .
$$

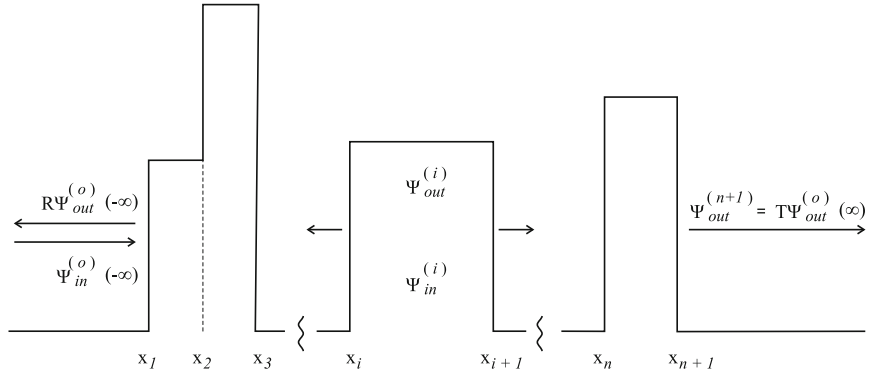

Fig. 1. System of barriers and potential steps according to quantum mechanics. $\Psi_{\text {in }}^{(0)}(-\infty)$ is incoming beam, $R \Psi_{\text {out }}^{(0)}(-\infty)$ reflected wave, $T \Psi_{\text {out }}^{(0)}(\infty)$ transmitted wave, $\Psi_{\text {in } / \text { out }}^{(0)}(x)$ underbarrier inward and outward wavefunction.

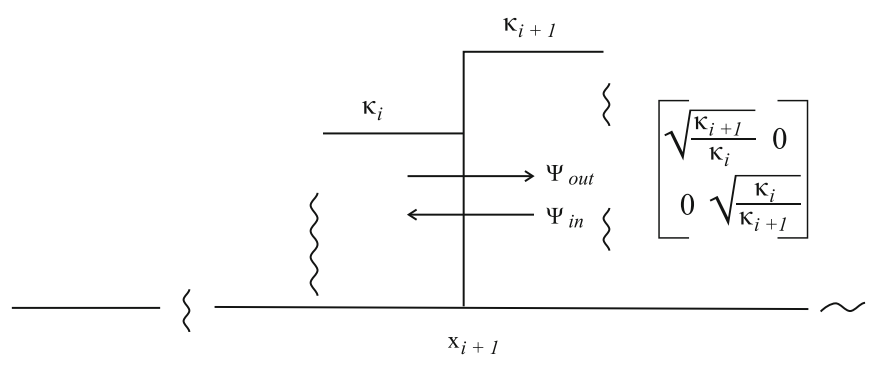

Fig. 2. The potential $i+1$ step, described by unimodular diagonal c-number matrix, $\kappa_{i}, \kappa_{i+1}$ are reduced barrier heights.

Multiplication of the $\mathcal{I}_{j+1}$ matrix and the matrices $W$ constitutes the total transition matrix i.e. one-step matrix through $j+1$ interface

$$
M_{j+1, j}^{(1)}\left(x_{j+1}\right)=\left[W^{(j+1)}\left(x_{j+1}\right)\right]^{-1} \mathcal{I}_{j+1}^{-1} W^{(j)}\left(x_{j+1}\right)
$$

$W$ is basic solution matrix in adjoining layers i.e. $j, j+1$, and its determinant $\operatorname{det}[W]$ is wronskian.

The transmission through two successive interfaces, what is equivalent to scattering on the barrier, is given by product of two one-step matrices

$$
\begin{aligned}
M_{j+2, j}^{(2)}( & \left.x_{j+2}, x_{j+1}\right) \\
= & {\left[W^{(j+2)}\left(x_{j+2}\right)\right]^{-1} \mathcal{I}_{j+2}^{-1} W^{(j+1)}\left(x_{j+2}\right) } \\
& \times\left[W^{(j+1)}\left(x_{j+1}\right)\right]^{-1} \mathcal{I}_{j+1}^{-1} W^{(j)}\left(x_{j+1}\right) .
\end{aligned}
$$

Alike we build the transmission $M$ for $n$ interfaces

$$
M_{n+1,0}\left(x_{n+1}, x_{1}\right)=\prod_{j=0}^{n} M_{j+1, j}^{(1)}\left(x_{j+1}\right)=M_{f, i n} .
$$

In Sprung et al. paper [13] authors introduce transmission matrices $W$ and $M$ both related by similarity transformation and composed from two independent solutions of the Schrödinger equation. Our method is different, matrix $M$ is not similar to $W$ and is not restricted to periodical systems. Our $M$ for one interface is given by equation (10). In case of two interfaces (two potential steps) is given by (11), 
and in case of multistep (or many interfaces) superposition $M$ is given by (12) as it is shown in Figure 1.

To show relation between equations (5) and (11) we rewrite the last one with help of wronskians and identity matrix $I=U^{-1} U$ as:

$$
\begin{aligned}
M_{j+2, j}^{(2)}\left(x_{j+2}, x_{j+1}\right)= & {\left[\Phi_{\text {in out }}^{(j+2)}\left(x_{j+2}\right)\right]^{-1} U^{-1} \mathcal{I}_{j+2}^{-1} U } \\
& \times \Phi_{\text {in out }}^{(j+1)}\left(x_{j+2}\right)\left[\Phi_{\text {in }, \text { out }}^{(j+1)}\left(x_{j+1}\right)\right]^{-1} \\
& \times U^{-1} \mathcal{I}_{j+1}^{-1} U \Phi_{\text {in out }}^{(j)}\left(x_{j+1}\right) .
\end{aligned}
$$

This equation without external matrices $\left[\Phi^{(j+2)}\right]$ and $\left[\Phi^{(j)}\right]$ has form of equation (5) where $U^{-1} \mathcal{I}_{2}^{-1} U$ is equivalent to

$$
\left[\begin{array}{cc}
\frac{1}{T_{2}^{*}} & -\frac{R_{2}^{*}}{T_{2}^{*}} \\
-\frac{R_{2}}{T_{2}} & \frac{1}{T_{2}}
\end{array}\right]
$$

while $U^{-1} \mathcal{I}_{1}^{-1} U$ to

$$
\left[\begin{array}{cc}
\frac{1}{T_{1}^{*}} & -\frac{R_{1}^{*}}{T_{1}^{*}} \\
-\frac{R_{1}}{T_{1}} & \frac{1}{T_{1}}
\end{array}\right] .
$$

The matrix

$$
\Phi_{\text {in }, \text { out }}^{(1)}\left(x_{2}\right)\left[\Phi_{\text {in out }}^{(1)}\left(x_{1}\right)\right]^{-1} \equiv \Phi_{j, k}
$$

in case of plane waves represents diagonal middle matrix in equation (5).

Sprung analysis can not be generalized to any barrier system. But it is particular case of our calculation when we deal with periodic barrier system and we use plane waves.

In optics the symmetric barrier is represented by external layers with the same properties $\left(n_{0}=n_{2}\right)$ where the middle layer has refraction index $n_{1}<\min \left(n_{0}, n_{2}\right)$. In that case, from the particular monodromy matrix (5), we get total reflection probability $|R|^{2}$ (cf. Eq. (8)) expressed as function of reflection probability from single interface $|r|^{2}$ if we assume that $\left|R_{1}\right|^{2}=\left|R_{2}\right|^{2}=|r|^{2}$ and $z=\exp (-\kappa L)$

$$
\begin{aligned}
|R|^{2} & =\frac{|r|^{2}\left(1-e^{-2 \kappa L}\right)\left(1-e^{-2 \kappa L}\right)}{\left(|r|^{2} e^{-2 \kappa L}-1\right)\left(|r|^{2} e^{-2 \kappa L}-1\right)} \\
& =\frac{|r|^{2}\left(1-z^{2}\right)^{2}}{\left(|r|^{2} z^{2}-1\right)^{2}} .
\end{aligned}
$$

The phase increment $\kappa L$ of the under-barrier wavefunction is calculated according to optics rules. In optical tunneling

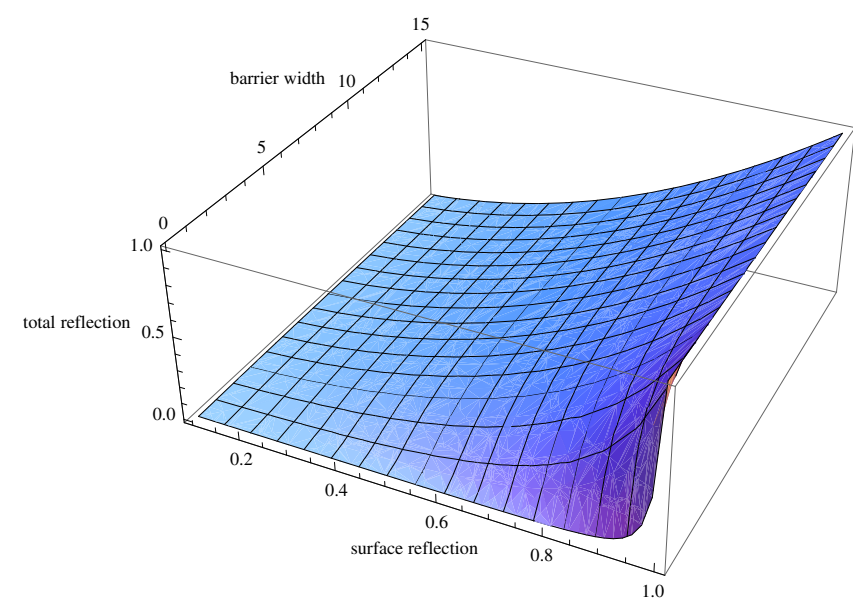

Fig. 3. Total reflection from three layer system according to equation (15) as function of the single surface reflection and barrier width, case $n_{0}=n_{2}$.

it is given by:

$$
\begin{aligned}
\kappa L=\kappa_{1} L & =k_{\text {vacuum }} L n_{1} \cos \vartheta_{1} \\
& =k_{\text {vacuum }} L n_{0} \sqrt{\sin ^{2} \vartheta_{0}-n_{1,0}^{2}} \\
& =k_{\text {vacuum }} L \sqrt{n_{0}^{2} \sin ^{2} \vartheta_{0}-n_{1}^{2}}
\end{aligned}
$$

where $k_{\text {vacuum }}=\frac{2 \pi}{\lambda_{\text {vacuum }}}$.

The dependence of the total reflection $|R|^{2}$ on the barrier width $L$ and on the middle layer reflection probability $|r|^{2}$ has been shown in Figure 3. The dependence on transmission $T$ according to condition $|R|^{2}+|T|^{2}=1$ can be imagined on the $|R|^{2}$ surface as certain curve starting from the right upper back corner $\left(|R|^{2}=1, L=15\right.$, $\left.|r|^{2}=1\right)$. In calculation the following parameters has been used: $n_{0}=1.4, n_{1}=1.0, \lambda_{\text {vacuum }}=7, \vartheta_{0}=54^{\circ}$. In case of visible light it can be accepted that one unit length is i.e. $100 \mathrm{~nm}$, and $\lambda$ has 7 units, then the maximum barrier width $L$ used in such calculations is 15 units.

Equation (15) for $|r|^{2}=1$ implies $|R|^{2}=1$. To our opinion that implication is source of the word 'frustrated' in FTIR. This crude approximation was used in references [14-16] and many others.

In case of three layer system with different refraction indexes $n_{0} \neq n_{1} \neq n_{2}$ corresponding to the reduced barrier height $\kappa_{1}$ with external wave numbers $\kappa_{0} \neq \kappa_{2}$, the monodromy theory equation (11) gives the following expression for tunneling-transmission probability through two step non-symmetric barrier:

$$
\begin{aligned}
\frac{1}{|T|^{2}} & =\frac{\left(\kappa_{2}+\kappa_{0}\right)^{2}}{4 \kappa_{2} \kappa_{0}}+\frac{\left(\kappa_{1}^{2}-\kappa_{0}^{2}\right)\left(\kappa_{1}^{2}-\kappa_{2}^{2}\right)}{4 \kappa_{1}^{2} \kappa_{2} \kappa_{0}} \sin ^{2}\left(\kappa_{1} L\right) \\
& =\beta+\alpha \sin ^{2}\left(\kappa_{1} L\right) .
\end{aligned}
$$

It is impossible to get this formula from equation (5) without quantum tunneling theory. 
The more general form of tunneling-transmission amplitude is:

$$
\begin{aligned}
\frac{1}{T}= & \frac{1}{2}\left(\sqrt{\frac{\kappa_{0}}{\kappa_{2}}}+\sqrt{\frac{\kappa_{2}}{\kappa_{0}}}\right) \frac{\left(\Phi_{11}+\Phi_{22}\right)}{2} \\
& +\frac{1}{2}\left(\sqrt{\frac{\kappa_{0}}{\kappa_{2}}}-\sqrt{\frac{\kappa_{2}}{\kappa_{0}}}\right) \frac{\left(\Phi_{12}+\Phi_{21}\right)}{2} \\
& +\frac{1}{2}\left(\sqrt{\frac{\kappa_{1}^{2}}{\kappa_{2} \kappa_{0}}}+\sqrt{\frac{\kappa_{2} \kappa_{0}}{\kappa_{1}^{2}}}\right) \frac{\left(\Phi_{22}-\Phi_{11}\right)}{2} \\
& +\frac{1}{2}\left(\sqrt{\frac{\kappa_{1}^{2}}{\kappa_{2} \kappa_{0}}}-\sqrt{\frac{\kappa_{2} \kappa_{0}}{\kappa_{1}^{2}}}\right) \frac{\left(\Phi_{12}-\Phi_{21}\right)}{2}
\end{aligned}
$$

where $\Phi_{j k}$ are complex quantities and are given by equation (14).

To convert the Schrödinger equation result into Maxwell equation we assume the following notation: $N=\frac{n_{0}}{n_{1}}, n=\frac{n_{2}}{n_{0}}, \mu_{1,0}=\frac{\mu_{1}}{\mu_{0}}, \varepsilon_{1,0}=\frac{\varepsilon_{1}}{\varepsilon_{0}}$ or more generally $n_{j, k}=\frac{n_{j}}{n_{k}}, \mu_{j, k}=\frac{\mu_{j}}{\mu_{k}}, \varepsilon_{j, k}=\frac{\varepsilon_{j}}{\varepsilon_{k}}$.

The symbols $N$ and $n$ are chosen to be compatible with Court and von Willisen [8] paper.

When we want to get from equation (16) an equivalent formula valid in optics, we must applied the following substitutions: (cf. Born and Wolf Eqs. (1.6-38,40)) $\kappa_{j} \Rightarrow p_{j}$ or $\kappa_{j} \Rightarrow q_{j}$ and the following relations:

- Substitution for TE wave:

$$
\kappa_{j} \Rightarrow p_{j}=\sqrt{\frac{\varepsilon_{j}}{\mu_{j}}} \cos \vartheta_{j} .
$$

When the Snell law is used, we get:

$$
\begin{aligned}
p_{1} & =\sqrt{\frac{\varepsilon_{1}}{\mu_{1}}} \cos \vartheta_{1}=\frac{1}{\mu_{1}} \sqrt{n_{1}^{2}-n_{0}^{2} \sin ^{2} \vartheta_{0}} \\
& =i \frac{n_{1}}{\mu_{1}} \sqrt{N^{2} \sin ^{2} \vartheta_{0}-1} .
\end{aligned}
$$

We apply substitution to the symbols $\sigma_{j, k}=\frac{\kappa_{j}}{\kappa_{k}}$ in the transmission formula (16) when it is rewritten in terms of:

$$
\begin{aligned}
\sigma=\sigma_{1,0} & =\frac{\kappa_{1}}{\kappa_{0}} \Rightarrow \frac{p_{1}}{p_{0}}=\sigma_{1,0}^{T E}=\frac{\sqrt{\frac{\varepsilon_{1}}{\mu_{1}}} \cos \vartheta_{1}}{\sqrt{\frac{\varepsilon_{0}}{\mu_{0}}} \cos \vartheta_{0}} \\
= & \frac{i \sqrt{N^{2} \sin ^{2} \vartheta_{0}-1}}{\mu_{1,0} N \cos \vartheta_{0}}=\frac{i \sqrt{\sin ^{2} \vartheta_{0}-n_{1,0}^{2}}}{\mu_{1,0} \cos \vartheta_{0}} \\
\sigma_{1,2} & =\frac{\kappa_{1}}{\kappa_{2}} \Rightarrow \frac{p_{1}}{p_{2}}=\sigma_{1,2}^{T E}=\frac{i \sqrt{N^{2} \sin ^{2} \vartheta_{0}-1}}{\mu_{1,2} N \sqrt{n_{2,0}^{2}-\sin ^{2} \vartheta_{0}}} \\
& =\frac{i \sqrt{\sin ^{2} \vartheta_{0}-n_{1,0}^{2}}}{\mu_{1,2} \sqrt{n_{2,0}^{2}-\sin ^{2} \vartheta_{0}}}
\end{aligned}
$$

$p_{2}$ is calculated from the refraction condition between first and third layers when the middle layer width approaches zero:

$$
\cos \vartheta_{2}=\sqrt{1-\frac{n_{0}^{2}}{n_{2}^{2}} \sin ^{2} \vartheta_{0}}
$$

- Substitution for $T M$ wave:

$$
\begin{aligned}
\kappa_{j} \Rightarrow q_{j}=\sqrt{\frac{\mu_{j}}{\varepsilon_{j}}} \cos \vartheta_{j} \\
q_{1}=i \frac{n_{1}}{\varepsilon_{1}} \sqrt{N^{2} \sin ^{2} \vartheta_{0}-1} \\
\sigma=\sigma_{1,0}=\frac{\kappa_{1}}{\kappa_{0}} \Rightarrow \frac{q_{1}}{q_{0}}=\sigma_{1,0}^{T M}=\frac{\sqrt{\frac{\mu_{1}}{\varepsilon_{1}}} \cos \vartheta_{1}}{\sqrt{\frac{\mu_{0}}{\varepsilon_{0}}} \cos \vartheta_{0}} \\
=\frac{i \sqrt{N^{2} \sin ^{2} \vartheta_{0}-1}=\frac{i \sqrt{\sin ^{2} \vartheta_{0}-n_{1,0}^{2}}}{\varepsilon_{1,0} N \cos \vartheta_{0}}}{\sigma_{1,0} \cos \vartheta_{0}} \\
=\frac{\kappa_{1}}{\kappa_{2}} \Rightarrow \frac{q_{1}}{q_{2}}=\sigma_{1,2}^{T M}=\frac{i \sqrt{N^{2} \sin ^{2} \vartheta_{0}-1}}{\varepsilon_{1,2} N} \sqrt{n_{2,0}^{2}-\sin ^{2} \vartheta_{0}} \\
=\frac{\mu_{1,2} n_{2,0}^{2} i \sqrt{\sin ^{2} \vartheta_{0}-n_{1,0}^{2}}}{n_{1,0}^{2} \sqrt{n_{2,0}^{2}-\sin ^{2} \vartheta_{0}}}
\end{aligned}
$$

- the wave number in the middle layer is:

$$
\kappa_{1}=k_{\text {vacuum }} n_{1} \cos \vartheta_{1}=i \frac{2 \pi}{\lambda_{1}} \sqrt{N^{2} \sin ^{2} \vartheta_{0}-1} .
$$

When all these relations are applied to quantum expression (16) we get equivalent optical tunneling expressions: in case of $T E$ wave:

$$
\left.\frac{1}{|T|^{2}}\right|_{T E}=\beta_{T E}+\alpha_{T E} \sinh ^{2}\left(\left|\kappa_{1}\right| L\right)
$$

and in case of $T M$ wave:

$$
\left.\frac{1}{|T|^{2}}\right|_{T M}=\beta_{T M}+\alpha_{T M} \sinh ^{2}\left(\left|\kappa_{1}\right| L\right) .
$$

After simple calculations we find the coefficients $\beta_{T E}, \alpha_{T E}$

$$
\begin{aligned}
\beta_{T E}= & \frac{\left(\sqrt{\frac{\mu_{0}}{\mu_{2}}} \sqrt{n^{2}-\sin ^{2} \vartheta_{0}}+\sqrt{\frac{\mu_{2}}{\mu_{0}}} \cos \vartheta_{0}\right)^{2}}{4 \cos \vartheta_{0} \sqrt{n^{2}-\sin ^{2} \vartheta_{0}}} \\
\alpha_{T E}= & \frac{\mu_{0} \mu_{2}}{\mu_{1}^{2}} \frac{\left(N^{2} \sin ^{2} \vartheta_{0}+\frac{\mu_{1}^{2}}{\mu_{0}^{2}} N^{2} \cos ^{2} \vartheta_{0}-1\right)}{4 N^{2} \cos \vartheta_{0}\left(N^{2} \sin ^{2} \vartheta_{0}-1\right) \sqrt{n^{2}-\sin ^{2} \vartheta_{0}}} \\
& \times \frac{\left(N^{2} n^{2} \frac{\mu_{1}^{2}}{\mu_{2}^{2}}-1+\left(N^{2}-N^{2} \frac{\mu_{1}^{2}}{\mu_{2}^{2}}\right) \sin ^{2} \vartheta_{0}\right)}{4 N^{2} \cos \vartheta_{0}\left(N^{2} \sin ^{2} \vartheta_{0}-1\right) \sqrt{n^{2}-\sin ^{2} \vartheta_{0}}} .
\end{aligned}
$$


In order to get the coefficients $\beta_{T M}, \alpha_{T M}$ it is enough to permute $\mu$ and $\epsilon$ in the expressions for $T E$ coefficients

$$
\begin{aligned}
& \beta_{T M}= \frac{\left(\sqrt{\frac{\varepsilon_{0}}{\varepsilon_{2}}} \sqrt{n^{2}-\sin ^{2} \vartheta_{0}}+\sqrt{\frac{\varepsilon_{2}}{\varepsilon_{0}}} \cos \vartheta_{0}\right)^{2}}{4 \cos \vartheta_{0} \sqrt{n^{2}-\sin ^{2} \vartheta_{0}}} \\
&= \frac{\left(\sqrt{\frac{\mu_{2}}{\mu_{0}}} \sqrt{n^{2}-\sin ^{2} \vartheta_{0}}+\sqrt{\frac{\mu_{0}}{\mu_{2}}} n^{2} \cos \vartheta_{0}\right)^{2}}{4 n^{2} \cos \vartheta_{0} \sqrt{n^{2}-\sin ^{2} \vartheta_{0}}} \\
& \alpha_{T M}= \frac{\varepsilon_{0} \varepsilon_{2}}{\varepsilon_{1}^{2}} \frac{\left(N^{2} \sin ^{2} \vartheta_{0}+\frac{\varepsilon_{1}^{2}}{\varepsilon_{0}^{2}} N^{2} \cos ^{2} \vartheta_{0}-1\right)}{4 N^{2} \cos \vartheta_{0}\left(N^{2} \sin ^{2} \vartheta_{0}-1\right) \sqrt{n^{2}-\sin ^{2} \vartheta_{0}}} \\
&= \frac{\left(N^{2} n^{2} \frac{\varepsilon_{1}^{2}}{\varepsilon_{2}^{2}}-1+\left(N^{2}-N^{2} \frac{\varepsilon_{1}^{2}}{\varepsilon_{2}^{2}}\right) \sin ^{2} \vartheta_{0}\right)}{4 N^{2} \cos \vartheta_{0}\left(N^{2} \sin ^{2} \vartheta_{0}-1\right) \sqrt{n^{2}-\sin ^{2} \vartheta_{0}}} \\
& 4 N^{2} \cos \vartheta_{0}\left(N^{2} \sin ^{2} \vartheta_{0}-1\right) \sqrt{n^{2}-\sin ^{2} \vartheta_{0}}\left(\frac{\mu_{2}}{\mu_{1}}-\frac{\mu_{1}}{\mu_{2}} N^{2} n^{2}+\left(\frac{\mu_{1}}{\mu_{2}} N^{4} n^{2}-\frac{\mu_{2}}{\mu_{1}} N^{2}\right) \sin ^{2} \vartheta_{0}\right) \\
& 4 N^{2} \cos \vartheta_{0}\left(N^{2} \sin ^{2} \vartheta_{0}-1\right) \sqrt{n^{2}-\sin ^{2} \vartheta_{0}}
\end{aligned}
$$

in approximation $\mu_{j}=1$ it is easy to get the following relation:

$$
\begin{aligned}
\alpha_{T M}= & \alpha_{T E}\left[1-\left(N^{2}+1\right) \sin ^{2} \vartheta_{0}\right] \\
& \times\left[\left(N^{2}+\frac{1}{n^{2}}\right) \sin ^{2} \vartheta_{0}-1\right]
\end{aligned}
$$

being proved using another method in reference [8].

The (never published) equations (23)-(26) are the most general expressions for tunneling in three layer system.

\section{Goos-Hänchen shift}

Goos-Hänchen shift $[5,6]$ occurs in the three layer system when light beam, traveling in the first layer $n_{0}$ falls onto the interface point $a$ under an angle greater than critical $\left(\vartheta_{0} \geq \vartheta_{c}=\arcsin \left(n_{1} / n_{0}\right)\right)$, is reflected from an air slab $n_{1}$ and emerges at the different point $b$ into the first medium.

In previous chapter we have considered particle transmission in $x$ direction. Now we take the plane of incidence to be $(x, z)$ plane and $z$ being the direction of stratification and transmission. The $x$ axis is parallel to interface. The incoming flux inside the air slab can be split into two fluxes parallel and perpendicular to layers.

For the scattering on the barrier we define underbarrier wavefunctions as a sum of two basic solution $\psi^{(1)}(z)=C_{1} \Psi_{\text {in }}^{(1)}\left(\kappa_{1} z\right)+D_{1} \Psi_{\text {out }}^{(1)}\left(\kappa_{1} z\right)$. According to Smith [17] and Hauge and Støvneng [18] papers we define $\mathcal{D}_{0}^{d w e l l}$ as a certain under-barrier dwell distance. We try to find physical interpretation of that length.
The evanescent and anti-evanescent tunnelling (transmitted) wavefunctions are defined on $\overline{c e}$ interval in $z$ direction (see Fig. 8). In many application in optics the evanescent (exponentially decaying) waves are only used [19]. Neglecting anti-evanescent waves is typical approximation procedure.

The dwell distance for symmetric three layer system $\left(\kappa_{0}=\kappa_{2}\right.$ and $\left.\sigma=\sigma_{1,0}=\sigma_{1,2}\right)$ is:

$$
\begin{aligned}
\mathcal{D}_{0}^{\text {dwell }}= & \int_{c}^{e}\left|\psi^{(1)}(z)\right|^{2} d z \\
& \times|T|^{2} \int_{-L / 2}^{L / 2}\left(\cosh ^{2}\left(\kappa_{1}\left(z-\frac{L}{2}\right)\right)\right. \\
& \left.+\frac{1}{\sigma^{2}} \sinh ^{2}\left(\kappa_{1}\left(z-\frac{L}{2}\right)\right)\right) d z \\
= & \mathcal{D}_{0, L}+\mathcal{D}_{0,2}^{\text {dwell }}=\mathcal{D}_{0, L}+\frac{\mathcal{A}}{\kappa_{1} \sigma} \\
= & \frac{L}{2}\left(1-\frac{1}{\sigma^{2}}\right)|T|^{2} \\
& +\frac{1}{2}\left(\sigma+\frac{1}{\sigma}\right) \sinh \left(\kappa_{1} L\right) \frac{\cosh \left(\kappa_{1} L\right)}{\kappa_{1} \sigma}|T|^{2}
\end{aligned}
$$

$\mathcal{D}_{0}^{\text {dwell }}$ consists of two elements $\mathcal{D}_{0, L}$ and $\mathcal{D}_{0,2}^{\text {dwell }}$. First part i.e. $\mathcal{D}_{0, L}$, for transmission far above the barrier, goes into classical distance - width of the barrier. Second part $\mathcal{D}_{0,2}^{\text {dwell }}=\frac{\mathcal{A}}{\kappa_{1} \sigma}$ is constant and does not disappear when $L \rightarrow \infty$.

$\mathcal{A}$ is defined as:

$$
\begin{aligned}
\mathcal{A} & =\frac{1}{2}\left(\sigma+\frac{1}{\sigma}\right) \sinh \left(\kappa_{1} L\right) \cosh \left(\kappa_{1} L\right)|T|^{2} \\
& =\frac{1}{4}\left(\sigma+\frac{1}{\sigma}\right) \sinh \left(2 \kappa_{1} L\right)|T|^{2} .
\end{aligned}
$$

$\mathcal{D}_{0}^{\text {dwell }}$ is called the dwell distance under the barrier in analogy to the dwell time.

Similarly we can define the phase distance adequately to the phase time. $\mathcal{D}_{0}^{\text {phase }}=\frac{\partial \phi}{\partial \kappa}$ where $\phi$ is the reflected or transmitted wavefunction phase

$$
\begin{aligned}
\mathcal{D}_{0}^{\text {phase }} & =\mathcal{D}_{0, L}+\frac{1}{\kappa_{1}}\left(\sigma+\frac{1}{\sigma}\right) \mathcal{A} \\
& =\mathcal{D}_{0, L}+\frac{1}{2 \kappa_{1}}\left(\sigma+\frac{1}{\sigma}\right)^{2} \sinh \left(\kappa_{1} L\right) \cosh \left(\kappa_{1} L\right)|T|^{2} .
\end{aligned}
$$

So there is the following relation between both distances:

$$
\mathcal{D}_{0}^{\text {phase }}=\mathcal{D}_{0}^{\text {dwell }}+\frac{1}{k} \mathcal{A} .
$$

These definitions result from transferring time problem [17] to the length definition in quantum mechanics.

For both distances $|T|^{2}$ is tunneling probability. In case of symmetric barrier it is convenient to write that expression as:

$$
|T|^{2}=\frac{1}{\cosh ^{2}\left(\kappa_{1} L\right)\left[1+\frac{1}{4}\left(\sigma-\frac{1}{\sigma}\right)^{2} \tanh ^{2}\left(\kappa_{1} L\right)\right]} .
$$




$$
\begin{aligned}
\mathcal{D}_{0,2}^{d w e l l, T E} & =\frac{\lambda_{\text {vacuum }}}{\pi n_{0}\left(\sin ^{2} \vartheta_{0}-n_{1,0}^{2}\right)^{1 / 2}} \frac{\left(\mu_{1,0}^{2} \cos ^{2} \vartheta_{0}+\sin ^{2} \vartheta_{0}-n_{1,0}^{2}\right) \mu_{1,0}^{2} \cos ^{2} \vartheta_{0} \tanh \left(\kappa_{1} L\right)}{\left[4 \mu_{1,0}^{2} \cos ^{2} \vartheta_{0}\left(\sin ^{2} \vartheta_{0}-n_{1,0}^{2}\right)+\left(\sin ^{2} \vartheta_{0}-\mu_{1,0}^{2} \cos ^{2} \vartheta_{0}-n_{1,0}^{2}\right)^{2} \tanh ^{2}\left(\kappa_{1} L\right)\right]} \\
& \stackrel{\mu=1}{\longrightarrow} \frac{\lambda_{\text {vacuum }}}{\pi n_{0}\left(\sin ^{2} \vartheta_{0}-n_{1,0}^{2}\right)^{1 / 2}} \frac{\left(1-n_{1,0}^{2}\right) \cos ^{2} \vartheta_{0} \tanh \left(\kappa_{1} L\right)}{\left[4 \cos ^{2} \vartheta_{0}\left(\sin ^{2} \vartheta_{0}-n_{1,0}^{2}\right)+\left(-\cos 2 \vartheta_{0}-n_{1,0}^{2}\right)^{2} \tanh ^{2}\left(\kappa_{1} L\right)\right]}
\end{aligned}
$$

Then the second term in the dwell distance is:

$$
\mathcal{D}_{0,2}^{\text {dwell }}=\frac{1}{2 \kappa_{1}}\left(1+\frac{1}{\sigma^{2}}\right) \frac{\tanh \left(\kappa_{1} L\right)}{1+\frac{1}{4}\left(\sigma-\frac{1}{\sigma}\right)^{2} \tanh ^{2}\left(\kappa_{1} L\right)}
$$

in the limit $L \rightarrow \infty$ we get:

$$
\mathcal{D}_{0,2}^{\text {dwell }} \stackrel{L \rightarrow \infty}{\longrightarrow} \frac{1}{2 \kappa_{1} \sigma}\left(\sigma+\frac{1}{\sigma}\right) \frac{4}{\left(\sigma+\frac{1}{\sigma}\right)^{2}}=\frac{2}{\kappa_{1}} \frac{1}{\left(\sigma^{2}+1\right)} .
$$

Making the substitution $\sigma=\sigma_{1,0}^{T E}$ for the $T E$ wave i.e. in equation (17), we find the second $T E$ part of the dwell distance

$$
\text { see equation above. }
$$

In the limit of infinite gap:

$$
\begin{aligned}
\mathcal{D}_{0,2}^{d w e l l, T E} \stackrel{L \rightarrow \infty}{\longrightarrow} \frac{2}{\kappa_{1}} \frac{1}{\left(\sigma^{2}+1\right)}= \\
\quad \frac{\lambda_{\text {vacuum }}}{\pi} \frac{1}{\sqrt{n_{0}^{2} \sin ^{2} \vartheta_{0}-n_{1}^{2}}} \frac{\mu_{1,0}^{2} \cos ^{2} \vartheta_{0}}{\mu_{1,0}^{2} \cos ^{2} \vartheta_{0}+\sin ^{2} \vartheta_{0}-n_{1,0}^{2}} .
\end{aligned}
$$

This equation should be compared to Renard $d_{\perp}$ formula:

$$
d_{\perp}=\mathcal{D}_{\text {Renard }}^{T E}=\frac{\sin \vartheta_{0}}{\mu_{1,0}} \mathcal{D}_{0,2}^{\text {dwell }, T E}(L \rightarrow \infty)
$$

The first part of the dwell distance as well as of the phase distance is given by $\mathcal{D}_{0, L}$, and for $T E$ wave after substitution $\sigma=\sigma_{1,0}^{T E}$ according to (17)

$$
\begin{aligned}
\mathcal{D}_{0, L}^{T E}= & \left.\frac{L}{2}\left(1-\frac{1}{\sigma^{2}}\right) \frac{1}{\left[1+\frac{1}{4}\left(\sigma+\frac{1}{\sigma}\right)^{2} \sinh ^{2}\left(\kappa_{1} L\right)\right]}\right|_{T E} \\
= & \frac{L}{2} \frac{\sin ^{2} \vartheta_{0}-\mu_{1,0}^{2} \cos ^{2} \vartheta_{0}-n_{1,0}^{2}}{\sin ^{2} \vartheta_{0}-n_{1,0}^{2}} \\
& \times \frac{1}{1+\frac{\left(\sin ^{2} \vartheta_{0}+\mu_{1,0}^{2} \cos ^{2} \vartheta_{0}-n_{1,0}^{2}\right)^{2}}{4 \mu_{1,0}^{2} \cos ^{2} \vartheta_{0}\left(\sin ^{2} \vartheta_{0}-n_{1,0}^{2}\right)} \sinh ^{2}\left(\kappa_{1} L\right)} \\
\stackrel{\mu=1}{\longrightarrow} \frac{L}{2} \frac{\left(-\cos 2 \vartheta_{0}-n_{1,0}^{2}\right)}{\sin ^{2} \vartheta_{0}-n_{1,0}^{2}} & \\
& \times \frac{1+\frac{\left(1-n_{1,0}^{2}\right)^{2}}{4 \cos ^{2} \vartheta_{0}\left(\sin ^{2} \vartheta_{0}-n_{1,0}^{2}\right)} \sinh ^{2}\left(\kappa_{1} L\right)}{}
\end{aligned}
$$

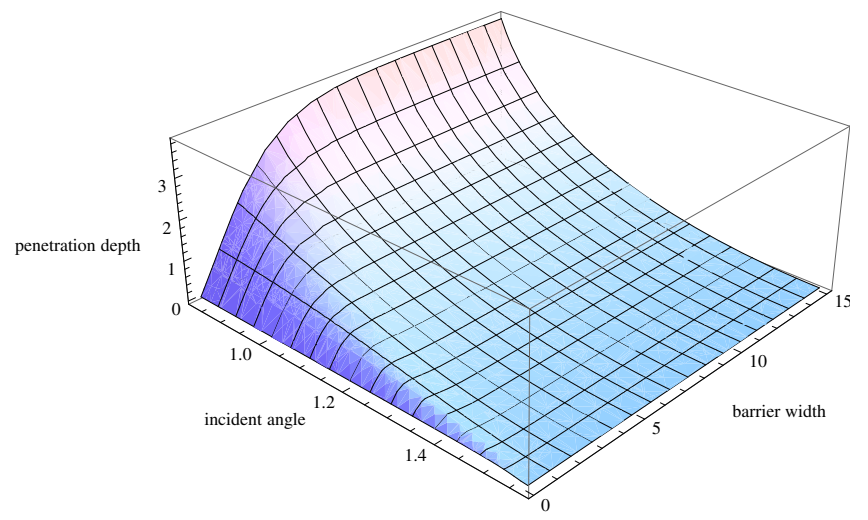

Fig. 4. $\mathcal{D}_{0}^{d w e l l, T E}$ as $T E$ penetration depth $\equiv T E$ dwell distance surface as first basic quantity to calculate Goos-Hänchen shift for $T E$ wave.

The total dwell distance $\mathcal{D}_{0}^{\text {dwell }}$ for the $T E$ wavefunction is shown in Figure 4.

Similarly for $T M$ : after substitution (19) into $\mathcal{D}_{0, L}$ and $\mathcal{D}_{0,2}^{\text {dwell }}$ or after replacement $\mu_{j, k} \rightarrow \varepsilon_{j, k}$ in $\mathcal{D}_{0, L}^{T E}$ we get formulas for $T M$ waves. It is comfortable to express all permittivities in formulas in term of refraction indexes and magnetic permeabilities with help of: $\mu_{j, k} \rightarrow \varepsilon_{j, k}=$ $n_{j, k}^{2} / \mu_{j, k}$

$$
\begin{aligned}
\mathcal{D}_{0, L}^{T M} & =\left.\frac{L}{2}\left(1-\frac{1}{\sigma^{2}}\right) \frac{1}{\left[1+\frac{1}{4}\left(\sigma+\frac{1}{\sigma}\right)^{2} \sinh ^{2}\left(\kappa_{1} L\right)\right]}\right|_{T M} \\
& =\frac{L}{2} \frac{\mu_{1,0}^{2} \sin ^{2} \vartheta_{0}-n_{1,0}^{4} \cos ^{2} \vartheta_{0}-\mu_{1,0}^{2} n_{1,0}^{2}}{\left(\sin ^{2} \vartheta_{0}-n_{1,0}^{2}\right)} \\
\times & \frac{1}{\mu_{1,0}^{2}+\frac{\left(\mu_{1,0}^{2} \sin ^{2} \vartheta_{0}+n_{1,0}^{4} \cos ^{2} \vartheta_{0}-\mu_{1,0}^{2} n_{1,0}^{2}\right)^{2}}{4 n_{1,0}^{4} \cos ^{2} \vartheta_{0}\left(\sin ^{2} \vartheta_{0}-n_{1,0}^{2}\right)} \sinh ^{2}\left(\kappa_{1} L\right)} \\
\mathcal{D}_{0, L}^{T M} & \stackrel{\mu=1}{\longrightarrow} \frac{\frac{L}{2} \frac{\left(\sin ^{2} \vartheta_{0}-n_{1,0}^{4} \cos ^{2} \vartheta_{0}-n_{1,0}^{2}\right)}{\sin ^{2} \vartheta_{0}-n_{1,0}^{2}}}{1+\frac{\left(\sin ^{2} \vartheta_{0}+n_{1,0}^{4} \cos ^{2} \vartheta_{0}-n_{1,0}^{2}\right)^{2}}{4 n_{1,0}^{4} \cos ^{2} \vartheta_{0}\left(\sin ^{2} \vartheta_{0}-n_{1,0}^{2}\right)} \sinh ^{2}\left(\kappa_{1} L\right)}
\end{aligned}
$$




$$
\begin{aligned}
\mathcal{D}_{0,2}^{\text {dwell }, T M} & =\frac{\lambda_{\text {vacuum }}}{\pi n_{0}\left(\sin ^{2} \vartheta_{0}-n_{1,0}^{2}\right)^{1 / 2}} \frac{\left(\varepsilon_{1,0}^{2} \cos ^{2} \vartheta_{0}+\sin ^{2} \vartheta_{0}-n_{1,0}^{2}\right) \varepsilon_{1,0}^{2} \cos ^{2} \vartheta_{0} \tanh \left(\kappa_{1} L\right)}{\left[4 \varepsilon_{1,0}^{2} g\left(\vartheta_{0}\right)+\left(\sin ^{2} \vartheta_{0}-\varepsilon_{1,0}^{2} \cos ^{2} \vartheta_{0}-n_{1,0}^{2}\right)^{2} \tanh ^{2}\left(\kappa_{1} L\right)\right]} \\
& =\frac{\lambda_{\text {vacuum }}}{\pi n_{0}\left(\sin ^{2} \vartheta_{0}-n_{1,0}^{2}\right)^{1 / 2}} \frac{\left(n_{1,0}^{4} \cos ^{2} \vartheta_{0}+\mu_{1,0}^{2}\left(\sin ^{2} \vartheta_{0}-n_{1,0}^{2}\right)\right) n_{1,0}^{4} \cos ^{2} \vartheta_{0} \tanh \left(\kappa_{1} L\right)}{\left[4 \mu_{1,0}^{2} n_{1,0}^{4} g\left(\vartheta_{0}\right)+\left(\mu_{1,0}^{2}\left(\sin ^{2} \vartheta_{0}-n_{1,0}^{2}\right)-n_{1,0}^{4} \cos ^{2} \vartheta_{0}\right)^{2} \tanh ^{2}\left(\kappa_{1} L\right)\right]} \\
& \stackrel{\mu=1}{\longrightarrow} \frac{\lambda_{\text {vacuum }}}{\pi n_{0}\left(\sin ^{2} \vartheta_{0}-n_{1,0}^{2}\right)^{1 / 2}} \frac{\left(n_{1,0}^{4} \cos ^{2} \vartheta_{0}+\left(\sin ^{2} \vartheta_{0}-n_{1,0}^{2}\right)\right) n_{1,0}^{4} \cos ^{2} \vartheta_{0} \tanh \left(\kappa_{1} L\right)}{\left[4 n_{1,0}^{4} g\left(\vartheta_{0}\right)+\left(\left(\sin ^{2} \vartheta_{0}-n_{1,0}^{2}\right)-n_{1,0}^{4} \cos ^{2} \vartheta_{0}\right)^{2} \tanh ^{2}\left(\kappa_{1} L\right)\right]}
\end{aligned}
$$

see equation above

where we put $g\left(\vartheta_{0}\right)=\cos ^{2} \vartheta_{0}\left(\sin ^{2} \vartheta_{0}-n_{1,0}^{2}\right)$.

In the limit of infinite gap for $T M$ :

$$
\begin{aligned}
\mathcal{D}_{0,2}^{\text {dwell }, T M} \stackrel{L \rightarrow \infty}{\longrightarrow} \frac{2}{\kappa_{1}} \frac{1}{\left(\sigma^{2}+1\right)} & \frac{\lambda_{\text {vacuum }}}{\pi} \frac{1}{\sqrt{n_{0}^{2} \sin ^{2} \vartheta_{0}-n_{1}^{2}}} \frac{\varepsilon_{1,0}^{2} \cos ^{2} \vartheta_{0}}{\varepsilon_{1,0}^{2} \cos ^{2} \vartheta_{0}+\sin ^{2} \vartheta_{0}-n_{1,0}^{2}} \\
= & \frac{\lambda_{\text {vacuum }}}{\pi} \frac{1}{\sqrt{n_{0}^{2} \sin ^{2} \vartheta_{0}-n_{1}^{2}}} \\
& \times \frac{n_{1,0}^{4} \cos ^{2} \vartheta_{0}}{n_{1,0}^{4} \cos ^{2} \vartheta_{0}+\mu_{1,0}^{2}\left(\sin ^{2} \vartheta_{0}-n_{1,0}^{2}\right)} .
\end{aligned}
$$

Comparing to Renard $d_{\|}$we get the following relation:

$$
d_{\|}=\mathcal{D}_{\text {Renard }}^{T M}=\frac{\sin \vartheta_{0}}{\varepsilon_{1,0}} \mathcal{D}_{0,2}^{d w e l l, T M}(L \rightarrow \infty) .
$$

The total dwell distance $\mathcal{D}_{0}^{d w e l l, T E}=\mathcal{D}_{0, L}^{T E}+\mathcal{D}_{0,2}^{d w e l l, T E}$ for the $T E$ and $\mu_{j}=1$ is shown in Figure 4 for the following parameters $n_{0}=n_{2}=1.5, n_{1}=1.0, \lambda_{\text {vacuum }}=7, \vartheta_{0} \in$ $\left(\vartheta_{c}, \pi / 2\right), L \in(0,15)$.

$\mathcal{D}_{0}^{d w e l l, T M}$ is shown in Figure 5.

In case of the phase distance:

$$
\begin{aligned}
& \mathcal{D}_{0,2}^{\text {phase }, T E}=\left.\frac{1}{2 \kappa_{1}}\left(\sigma+\frac{1}{\sigma}\right)^{2} \frac{\tanh \left(\kappa_{1} L\right)}{1+\frac{1}{4}\left(\sigma-\frac{1}{\sigma}\right)^{2} \tanh ^{2}\left(\kappa_{1} L\right)}\right|_{T E} \\
& =\frac{\lambda_{\text {vacuum }}}{\pi n_{0}\left(\sin ^{2} \vartheta_{0}-n_{1,0}^{2}\right)^{1 / 2}} \\
& \times \frac{\left(\mu_{1,0}^{2} \cos ^{2} \vartheta_{0}+\sin ^{2} \vartheta_{0}-n_{1,0}^{2}\right)^{2} \tanh \left(\kappa_{1} L\right)}{\left[4 \mu_{1,0}^{2} g\left(\vartheta_{0}\right)+\left(\sin ^{2} \vartheta_{0}-\mu_{1,0}^{2} \cos ^{2} \vartheta_{0}-n_{1,0}^{2}\right)^{2} \tanh ^{2}\left(\kappa_{1} L\right)\right]} \\
& \stackrel{\mu=1}{\longrightarrow} \frac{\lambda_{\text {vacuum }}}{\pi n_{0}\left(\sin ^{2} \vartheta_{0}-n_{1,0}^{2}\right)^{1 / 2}} \\
& \quad \times \frac{\left(1-n_{1,0}^{2}\right)^{2} \tanh ^{2}\left(\kappa_{1} L\right)}{\left[4 g\left(\vartheta_{0}\right)+\left(-\cos 2 \vartheta_{0}-n_{1,0}^{2}\right)^{2} \tanh ^{2}\left(\kappa_{1} L\right)\right]} .
\end{aligned}
$$

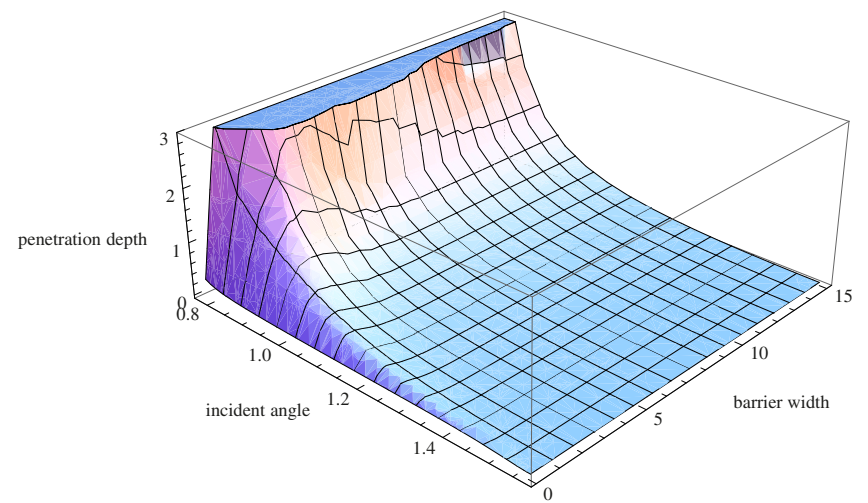

Fig. 5. $\mathcal{D}_{0}^{\text {dwell }, T M}$ as $T M$ penetration depth $\equiv T M$ dwell distance surface as first basic quantity to calculate Goos-Hänchen shift for $T M$ wave.

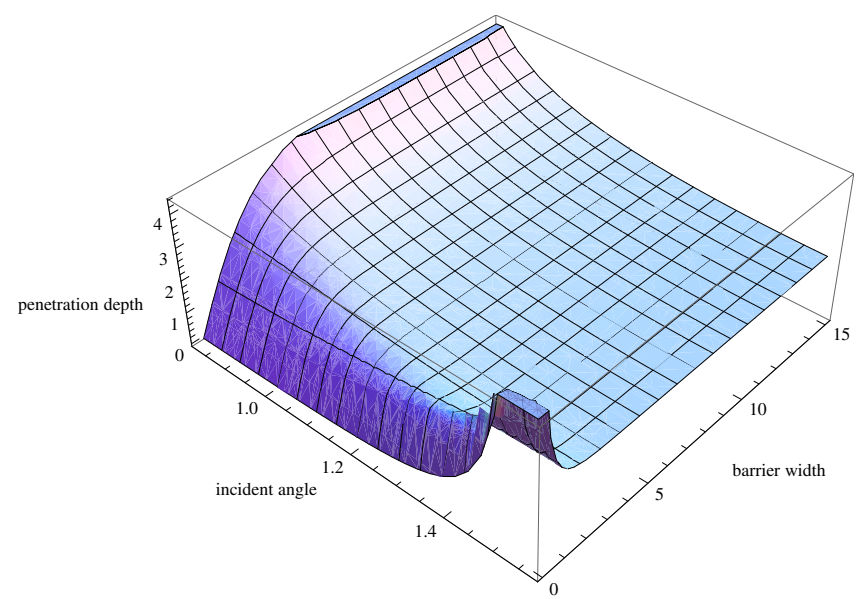

Fig. 6. $\mathcal{D}_{0}^{\text {phase }, T E}$ as $T E$ penetration depth $\equiv$ phase distance surface as second basic quantity to calculate Goos-Hänchen shift for $T E$ wave.

The total phase distance for the $T E$ wave is the sum of two sub-distances $\mathcal{D}_{0}^{\text {phase }, T E}=\mathcal{D}_{0, L}^{T E}+\mathcal{D}_{0,2}^{\text {phase }, T E}$ and is shown in Figure 6.

That distance does not disappear for the infinite gap and for grazing angles as well as tangent beam $\vartheta_{0} \rightarrow \pi / 2$. It is Hartman effect [20] for the phase distance. Only experiment can answer the question if $\mathrm{G}-\mathrm{H}$ in that limit tends to zero or remains finite. 


$$
\begin{aligned}
D_{0,2}^{\text {phase }, T M} & =\frac{\lambda_{\text {vacuum }}}{\pi n_{0}\left(\sin ^{2} \vartheta_{0}-n_{1,0}^{2}\right)^{1 / 2}} \frac{\left(n_{1,0}^{4} \cos ^{2} \vartheta_{0}+\mu_{1,0}^{2}\left(\sin ^{2} \vartheta_{0}-n_{1,0}^{2}\right)\right)^{2} \tanh \left(\kappa_{1} L\right)}{\left[4 \mu_{1,0}^{2} n_{1,0}^{4} g\left(\vartheta_{0}\right)+\left(\mu_{1,0}^{2}\left(\sin ^{2} \vartheta_{0}-n_{1,0}^{2}\right)-n_{1,0}^{4} \cos ^{2} \vartheta_{0}\right)^{2} \tanh ^{2}\left(\kappa_{1} L\right)\right]} \\
& \stackrel{\mu=1}{\longrightarrow} \frac{\lambda_{\text {vacuum }}}{\pi n_{0}\left(\sin ^{2} \vartheta_{0}-n_{1,0}^{2}\right)^{1 / 2}} \frac{\left(n_{1,0}^{4} \cos ^{2} \vartheta_{0}+\sin ^{2} \vartheta_{0}-n_{1,0}^{2}\right)^{2} \tanh \left(\kappa_{1} L\right)}{\left[4 n_{1,0}^{4} g\left(\vartheta_{0}\right)+\left(\sin ^{2} \vartheta_{0}-n_{1,0}^{2}-n_{1,0}^{4} \cos ^{2} \vartheta_{0}\right)^{2} \tanh ^{2}\left(\kappa_{1} L\right)\right]}
\end{aligned}
$$

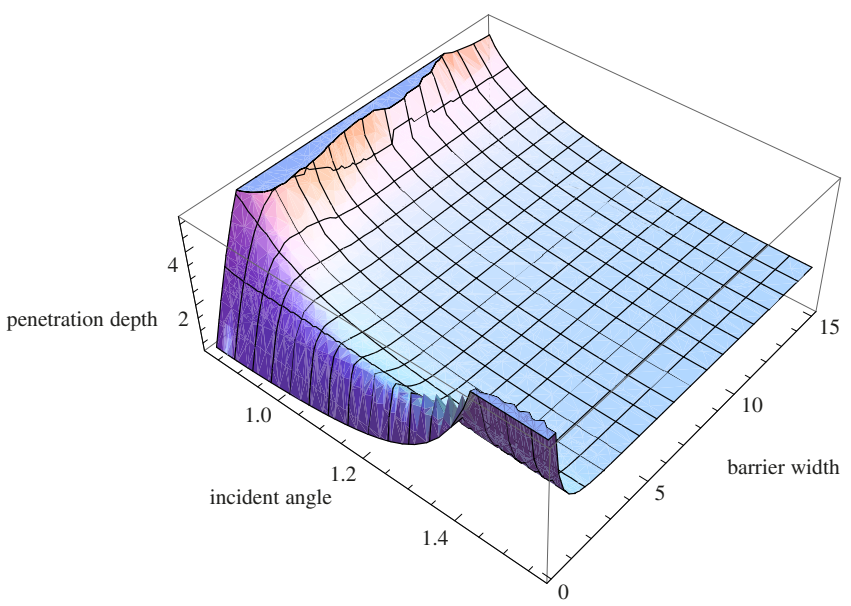

Fig. 7. $\mathcal{D}_{0}^{\text {phase }, T M}$ as $T M$ penetration depth $\equiv$ phase distance surface as second basic quantity to calculate Goos-Hänchen shift for $T M$ wave.

Similarly after substitution $\sigma=\sigma_{1,0}^{T M}$ we get $\mathcal{D}_{0}^{\text {phase }, T M}=\mathcal{D}_{0, L}^{T M}+\mathcal{D}_{0,2}^{\text {phase }, T M}$ where

see equation above

$\mathcal{D}_{0}^{\text {phase, }}$ TM is shown in Figure 7.

In case of infinite gap we get:

$$
\begin{aligned}
\mathcal{D}_{0}^{\text {phase }} & \stackrel{L \rightarrow \infty}{\longrightarrow} \frac{1}{2 \kappa_{1}}\left(\sigma+\frac{1}{\sigma}\right)^{2} \frac{4}{\left(\sigma+\frac{1}{\sigma}\right)^{2}}=\frac{2}{\kappa_{1}} \\
& =\frac{\lambda_{\text {vacuum }}}{\pi} \frac{1}{\sqrt{n_{0}^{2} \sin ^{2} \vartheta_{0}-n_{1}^{2}}} .
\end{aligned}
$$

In that limit $\mathcal{D}_{0}^{\text {phase }}$ is the same for both $T E$ and $T M$ waves.

Equation (33) is a source of the earliest fits of the experimental G-H shift $\left(\mathcal{D}_{G H-\text { shift }}^{\text {empirical }} \approx \mathcal{D}_{0,2}^{\text {phase }}(L \rightarrow \infty)\right)$.

\section{The physical interpretation of $\mathcal{D}_{0}^{\text {phase }} \mathbf{i} \mathcal{D}_{0}^{\text {dwell }}$}

In scientific literature there is no consistent G-H theory and it does not fit the experimental data. The main problem from the theoretical point of view is to be able to define measurable quantum distances.

From the quantum mechanical point of view and interpretation of $(27)$, quantities $\mathcal{D}_{0}$ i.e. $\left(\mathcal{D}_{0}^{\text {dwell }}\right.$ or $\left.\mathcal{D}_{0}^{\text {phase }}\right)$

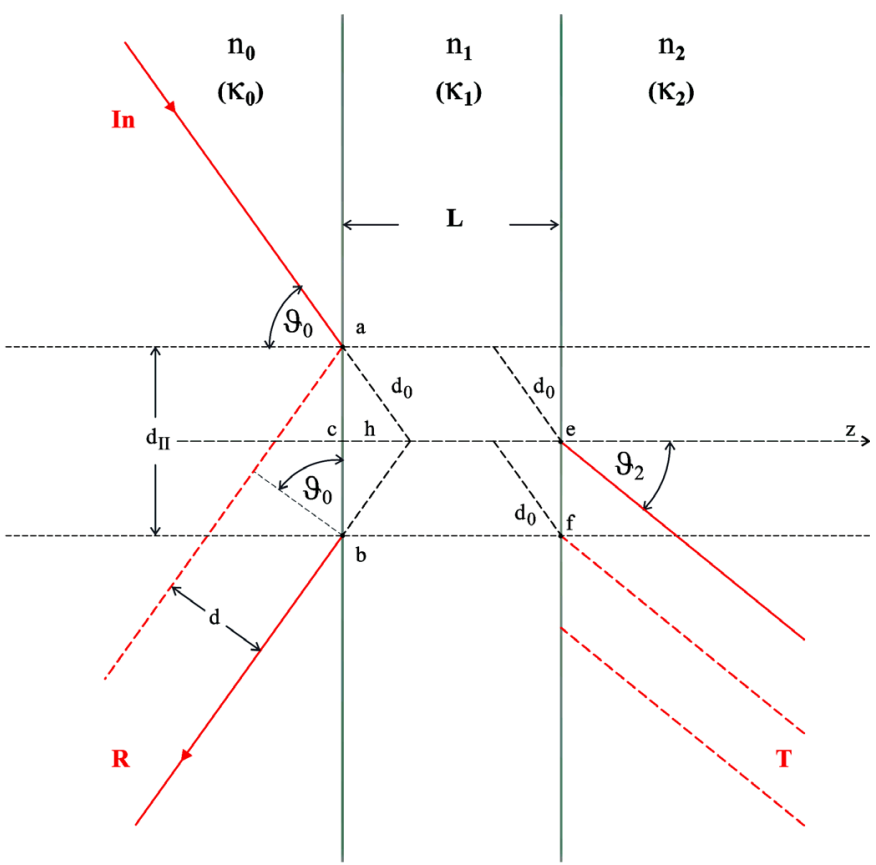

Fig. 8. The simplified geometry of the Goos-Hänchen shift. $\vartheta_{0}$ is the angle of incidence, $\vartheta_{2}$ - the angle of the transmitted beam, $R$ the reflected beam, $T$ the transmitted beam, $L$ width of the air slab, $d_{\|}$the distance between the point of incidence $a$ and the point $b$ where the beam emerges when reflected, $d_{0}$ the penetration distance, $h$ penetration depth, $d$ shift in reflected beam, $n_{0}, n_{1}, n_{2}$ the refraction indexes in subsequent layers.

should define certain barrier penetration depth. Both distances, as it results from Figures $4-7$ behave differently when $L \rightarrow \infty ; \vartheta_{0} \rightarrow \pi / 2$, the dwell distance goes to zero, the phase distance remains finite.

That distances should be related to quantities experimentally measured. The equations proportional to (29), (31) i.e. $\mathcal{D}_{0}^{\text {dwell }}(L \rightarrow \infty, \mu=1)$ were discussed in Renard [7] paper. Equation (33) i.e. $\mathcal{D}_{0}^{\text {phase }}(L \rightarrow \infty)$ was discarded as unrealistic but it result from the definition of phase distance.

The simplified geometry of Goos-Hänchen shift is shown in Figure 8. The incident photon behaves like a ball reflected from the springboard (i.e. elastic scattering in the potential field). We assume that the top of isosceles triangle built on $\overline{a b}$ interval is apparent reflected photon point. Distance $a b$ is dependent on the penetration 
depth $h$. Below we define quantities which should be related to dwell or phase distances:

$-d_{\|}$: the distance between points $a, b . d_{\|}=2 d_{0} \sin \left(\vartheta_{0}\right)$ - if the light beam slides on the interface, $d_{\|}$should be proportional to $\mathcal{D}_{0}^{\text {dwell }}$.

$-d_{0}$ : it is half of the real dwell distance. Such two intervals $d_{0}$ together with $d_{\|}$form isosceles triangle. Its height is $h$ (Of course it is a simple approximation because the light trajectory between $a$ and $b$ points remains unknown.).

- $h: d_{\|}=2 h \tan \left(\vartheta_{0}\right)-$ the dwell distance as (double) penetration depth.

From these quantities we calculate $d$ : the parallel shift of the reflected beam at point $b$ against possible reflection at point $a . d=2 d_{0} \sin \left(\vartheta_{0}\right) \cos \left(\vartheta_{0}\right)=d_{\|} \cos \left(\vartheta_{0}\right)=2 h \sin \left(\vartheta_{0}\right)$.

Only an experiment can answer the question, which quantity from $\left\{d_{0}, h, d_{\|}\right\}$is related to $\mathcal{D}_{0}$ if the simplified geometry is working.

Heibel et al. in their paper [21] suggest following choice $d_{\|}=\mathcal{D}_{0}^{\text {phase }}$. It is also possible that $\mathcal{D}_{0}$ consists of one or two intervals $d_{0}$ or one or two intervals $h$. Unfortunately there is no data showing where tunneling photon appears. Available are only data from tunneling time TE, TM measurements as those in reference [22] based on complex transmission time (That time consists of the real phase time and the imaginary so called loss-time.). For asymmetric systems phases (times) related to reflection and transmission can be different.

Renard's calculations suggest that $2 h$ is equal to $\mathcal{D}_{0}^{\text {dwell }}$. If so, the above geometric formulas for the infinite gap differ by the magnetic permeability ratio (for TE wave, see Eq. (30)) and by the permitivity ratio (for $T M$ wave, see Eq. (32)) from Renard's expressions for $d$ shift. Renard's geometry dependent method shows he integrates the Maxwell equation between $a$ and $b$ points. We integrate evanescent and anti-evanescent waves between $c$ and $e$ points. Such choice forbids negative $\mathrm{G}-\mathrm{H}$ shift found in reference [23]. Renard and Artmann described two physically different situations. Artmann calculations are the limit of $\mathcal{D}_{0}^{\text {phase }}$ while Renard's the limit of $\mathcal{D}_{0}^{\text {dwell }}$.

According to Renard the surface wave travels from point $a$ to point $b$. This wave is not standing, as is often stated. It travels in the $x$ direction in the incident plane, parallel to the interface 1 . His main assumption to calculate the G-H shift is equality of the time average energy flux for the reflected wave across a strip whose width is $d$ with the time average energy flux generated by the surface wave in the entire medium of lower index. To generalize that point of view, we consider the flow of energy through the barrier described by the complex Poynting vector. Next we analyse the flux conservation in the incident plane by means of the planar variant of the divergence theorem.

A few words about the dwell time and the phase time. It is generally accepted [18] that the dwell time $\tau_{D}$ is a measure of the time averaged over all scattering channels spent by a particle in a region of space. It can be defined locally for arbitrary points $x_{1}$ and $x_{2}$. The phase time seems to be asymptotic in character result of the extrapolating procedure to fit that time to scattering region inside its boundary i.e. in one dimension between points $x_{1}, x_{2}$, or between points $c, e$, in two dimension between the interval $\overline{a b}$ and point $e$ or between the interval $\overline{a b}$ and the interval which includes $\overline{f, e}$. But such corollary time definitions cause problems when such ones are being applied to distances. The calculations show that dwell distance is related to the reflection channel while the phase distance contains the dwell distance and another part $(\mathcal{A})$ which is responsible for the transmission (tunneling) channel.

To compare properties of the photon TE, TM wavefunction with one dimensional Schrödinger wavefunction the method of separation of variables is applied to the photon wavefunction written below:

$$
\begin{aligned}
\boldsymbol{F}(\boldsymbol{r}, t) & =\left(\frac{\boldsymbol{D}(\boldsymbol{r}, t)}{\sqrt{2 \varepsilon_{0}}}+i \frac{\boldsymbol{B}(\boldsymbol{r}, t)}{\sqrt{2 \mu_{0}}}\right) \\
|\boldsymbol{F}(x, y, z)|^{2} & \left|\frac{\sum_{j} D_{j}(x, y) \hat{e}_{j} U_{j}(z)}{\sqrt{2 \varepsilon_{0}}}+i \frac{\sum_{j} B_{j}(x, y) \hat{e}_{j} V_{j}(z)}{\sqrt{2 \mu_{0}}}\right|^{2} .
\end{aligned}
$$

Very interesting case is what we get if we integrate the photon $T E, T M$ separable wavefunctions over the incident plane between two interfaces. The result is:

$$
\begin{aligned}
\left.\int_{c}^{e} \int_{a}^{b}|\boldsymbol{F}(x, z)|^{2} d x d z\right|_{\substack{\text { TE,TM } \\
\text { photon } \\
\text { FTIR }}}=\frac{\partial \phi}{\partial k} \\
=\left.\int_{c}^{e}\left|\psi^{(1)}(z)\right|^{2} d z\right|_{\begin{array}{c}
\text { barrier } \\
\text { quantum } \\
\text { tunneling }
\end{array}}+\frac{\mathcal{A}}{k} .
\end{aligned}
$$

The photon dwell surface in that case is equal to particle phase distance.

This relation is also valid for tunneling through the waveguide. In the incident plane the Poynting vector $\mathcal{P}$ has two components, one in $x$ direction another in $z$ direction. There are at least two ways to calculate the change of flow inside the air gap. Directly, by integrating $\nabla_{x} \mathcal{P}_{x}+$ $\nabla_{z} \mathcal{P}_{z}$ over the rectangular surface, from the divergence theorem, or its variational version to evaluate the timeaverage stored energy.

In both methods the $x$ component is proportional to $\int^{e}\left|U_{y}(z)\right|^{2} d z$ while the $z$ component to $\mathcal{A}$.

We have come to the following conclusion:

The total phase $\phi$ contains the most general information, a certain global information about all (lossless) channels. From the total phase we can separate parts responsible for scattering in a given channel. It is possible to assign sub-phases to the reflection and tunnelling (scattering) channels. Such analysis shows that a certain part 
of the total phase $\phi$ defines the lateral shift therefore calculation using $\frac{\partial \phi}{\partial k_{\|}}$cannot be correct.

It is easy to construct the dwell distance $\mathcal{D}_{0}^{\text {dwell }}$ for three different layers with refraction indexes $n_{0} \neq n_{1} \neq n_{2}$ if we apply expression similarity steaming from monodromy

$$
\begin{aligned}
\mathcal{D}_{0}^{\text {dwell }}\left(n_{0} \neq n_{2}\right)= & \int_{c}^{e}\left|\psi^{(1)}(z)\right|^{2} d z \\
= & \frac{L}{2}\left(1-\frac{1}{\sigma_{f}^{2}}\right)|T|^{2} \\
& +\left(1+\frac{1}{\sigma_{f}^{2}}\right) \frac{\sinh \left(2 \kappa_{1} L\right)}{4 \kappa_{1}}|T|^{2} \\
= & \frac{L}{2}\left(1-\frac{1}{\sigma_{f}^{2}}\right)|T|^{2} \\
& +\frac{1}{2}\left(\sigma_{f}+\frac{1}{\sigma_{f}}\right) \sinh \left(\kappa_{1} L\right) \frac{\cosh \left(\kappa_{1} L\right)}{\kappa_{1} \sigma_{f}}|T|^{2}
\end{aligned}
$$

where $\sigma_{f}=\sigma_{1,2}^{T E}$ (see Eq. (18)) $\vee \sigma_{f}=\sigma_{1,2}^{T M}$ (see Eq. (20)), and $|T|^{2}$ is given by equation (16) or (21) for $T E$ wave and (22) for $T M$ wave.

Generalization is not so simple in case of the phase distance. For the asymmetric layers the reflection and tunneling amplitude phases are different.

In accordance to quantum mechanics we integrate Maxwell-Schrödinger equations in both direction perpendicular and parallel to layers (stratified media). This suggests that $\mathcal{D}_{0}^{\text {dwell }}$ in barrier tunneling should be related to the penetration depth which can not be measured directly. Simplified geometry transforms that depth into the observed $d_{\|}$distance. Such approximation is in agreement with previous calculations based on setup with infinite middle layer width. In reality the G-H shift should be calculated from $\mathcal{P}_{x}$ flow in accordance with the flux conservation.

$\mathcal{D}_{0}^{\text {phase }}$ remains finite even for the wide middle layer. In that limit the phase distance is the same for $T E$ as well as $T M$ waves. Time related to the phase distance called the phase time is base of superluminar considerations [24].
Physicists incline to conclusion about superiority of the phase time over the dwell one. Our G-H shift theory shows that $\mathcal{D}_{0}^{\text {dwell }}$ not $\mathcal{D}_{0}^{\text {phase }}$ has physical meaning. It seems now impossible to attach $\mathcal{D}_{0}^{\text {phase }}$ to any geometric distances.

\section{References}

1. E.E. Hall, Phys. Rev. 15, 73 (1902)

2. K. Försteling, Ann. Phys. 35, 745 (1937)

3. H. Arzelies, Ann. Phys. 1, 5 (1946)

4. P.J. Leurgans, A.F. Turner, J. Opt. Soc. Am. 37, 983 (1947)

5. F. Goos, H. Hänchen, Ann. Phys. 1, 333 (1947)

6. F. Goos, H. Hänchen, Ann. Phys. 5, 251 (1949)

7. R. Renard, J. Opt. Soc. Am. 54, 1190 (1964)

8. I.N. Court, F.K. von Willisen, Appl. Opt. 3, 719 (1964)

9. E.D. Rainville, in Intermediate Differential Equation (The MacMillan Company, New York, 1964), Chap. 4, § 25

10. M. Abramowitz, I.A. Stegun, in Handbook of Mathematical Functions (1964), Chap. 10, p. 14

11. V.I. Arnold, Geometric Methods in the Theory of Ordinary Differential Equations (Springer, 1987)

12. M. Born, E. Wolf, in Principles of Optics (Pergamon Press, 1975), Chap. 1.6

13. D.W.L. Sprung, H. Wu, J. Martorell, Am. J. Phys. 61, 1118 (1993)

14. S. Zhu, A.W. Yu, D. Hawley, R. Roy, Am. J. Phys. 54, 601 (1985)

15. Y. You et al., Am. J. Phys. 76, 224 (2008)

16. Z. Vörös, R. Johnsen, Am. J. Phys. 76, 746 (2008)

17. F.T. Smith, Phys. Rev. 118, 349 (1960)

18. E.H. Hauge, J.A. Støvneng, Rev. Mod. Phys. 61, 917 (1989)

19. Th. Martin, R. Landauer, Phys. Rev. A 45, 2611 (1992)

20. T.E. Hartman, J. Appl. Phys. 33, 3427 (1962)

21. A. Heibel, G. Nimtz, A.A. Stahlhofen, Phys. Rev. E 63, 047601 (2001)

22. Ph. Balcou, L. Dutriaux, Phys. Rev. Lett. 78, 851 (1997)

23. C.-F. Li, Phys. Rev. Lett. 91, 133903 (2003)

24. V.S. Olkhovsky, E. Recami, J. Jakiel, Phys. Rep. 398, 133 (2004)

Open Access This is an open access article distributed under the terms of the Creative Commons Attribution License (http://creativecommons.org/licenses/by/4.0), which permits unrestricted use, distribution, and reproduction in any medium, provided the original work is properly cited. 